\section{Reseña / Los caminos de la extensión \\ en Colombia}

Valenzuela Tovar, S. M. y del Campo Machado, R. M. (Comps) (2021). Los caminos de la Extensión en Colombia. Asociación Colombiana de Universidades (ASCUN). ISBN: 978-958-8481-49-4 e ISBN 978-9588481-50-0. Sitio de descarga gratuita: https://ascun.org.co/uploads/ default/networks/de5d7d6e717daa81d4dab9bede1db3c1.pdf

Por Ricardo Santiago Puca Molina

Escuela de Comunicación. Unidad Académica Río Gallegos. Universidad Nacional de la Patagonia Austral, Argentina. spucamolina@uarg.unpa.edu.ar
Ambiente y extensión universitaria / Reseñas (ㄷ)(1)(2)

En el libro Los caminos de la extensión en Colombia el lector va a hallar un producto de gran valor académico que da cuenta de la trayectoria de una de las funciones sustantivas de la educación superior en ese país. Retoma los objetivos de la tríada generada por la Universidad Nacional de La Pampa (Argentina) y conformada por los ejemplares Los caminos de la extensión universitaria argentina, Los caminos de la extensión en América Latina y el Caribe y Los caminos de la extensión en la Universidad Nacional de La Pampa, todos ellos con el espíritu presente del querido amigo y maestro Jorge Orlando Castro.

El prólogo es de Oscar Domínguez González, director ejecutivo de la Asociación Colombiana de Universidades (ASCUN) y la presentación está a cargo del Lic. Juan Carlos Molinas, vicepresidente de la Unión Latinoamericana de Extensión Universitaria (ULEU). En su texto se ven reflejadas 12 universidades de Colombia mediante la publicación de 14 artículos en los cuales participaron 30 autores.

Podemos encontrar distintas experiencias que retoman la política de extensión de la Red Nacional de Extensión Universitaria de ASCUN y observar cuáles son los lineamientos que se proponen con relación a las formas de hacer extensión en las universidades colombianas. Ellas son: Educación continua o continuada, Servicios de asesoría y consultorías, Servicios docente-asistenciales, Gestión de la innovación, Programas interdisciplinarios de extensión que integran formación e investigación, Prácticas y pasantías universitarias, Gestión cultural, Gestión de la relación con los graduados, y Voluntariado.

Esta obra impulsa a continuar desarrollando la extensión como política universitaria en Colombia con grandes cambios en la sociedad como objetivo. En cada una de las experiencias presentadas se van a encontrar aportes para lograr futuros ciudadanos comprometidos con sus propios territorios. También en varios capítulos del libro se analiza la posibilidad de articular las tres funciones del sistema universitario, de modo de potenciar la denominada integralidad de las funciones.

El libro está compuesto por seis capítulos dedicados a los Nodos o secciones del país. Ellos son: Nodo Centro (Cundinamarca, Boyacá, Meta, Tolima, Huila, Caquetá); Nodo Caribe (Guajira, César, Magdalena, Atlántico, Bolívar, Sucre, Córdoba y San Andrés); Nodo Occidente (Antioquia y Chocó); Nodo Oriente (Santander, Norte de Santander); Nodo Eje Cafetero (Caldas, Risaralda, Quindío); Nodo Sur Occidente (Valle de Cauca, Cauca, Nariño). En tanto, el séptimo capítulo plantea los retos nacionales. 
La Red Nacional de Extensión Universitaria de la ASCUN logró, con este texto, unir actores universitarios y distintas comunidades colombianas que dan cuenta de las acciones extensionistas desarrolladas. También es de destacar el ejercicio profundo de los antecedentes valiosísimos en materia de revisión bibliográfica. Considero relevante decir que las experiencias aquí plasmadas se constituyen en elementos para trabajar y reflexionar respecto de las producciones escritas sobre extensión universitaria.

Finalmente, es interesante el reconocimiento a la Red, que por muchos años propició encuentros y espacios de reflexión y análisis y que alcanza, en este libro, la consolidación y fortalecimiento de las Unidades de Extensión en las Universidades e Instituciones de Educación Superior en Colombia. Se trata de una contribución a la construcción grupal y colectiva desde el conocimiento y el reconocimiento de las prácticas extensionistas y desde las conceptualizaciones de esta función, pero también se estimula a la reflexión y a la producción de distintos especialistas que abordan a la extensión universitaria. 